\title{
Going Deeper in Cryo Electron Tomography with Neural Networks
}

\author{
Muyuan Chen ${ }^{1,2}$, Wei Dai ${ }^{2, \#}$, Stella Y. Sun ${ }^{2}$, Michael F. Schmid ${ }^{2}$, Wah Chiu ${ }^{2}$, Steven J. Ludtke ${ }^{2}$ \\ 1. Graduate Program in Structural and Computational Biology and Molecular Biophysics, Baylor \\ College of Medicine, Houston, TX \\ 2. Verna Marrs and McLean Department of Biochemistry and Molecular Biology, Baylor College of \\ Medicine, Houston, TX
}

Cellular Electron Cryotomography (CryoET) is a technique for studying the structure of interacting, dynamic complexes in their native cellular environment. Despite its rapid development recently, CryoET suffers from three major limiting factors: the very high noise levels, the missing wedge artifact due to the limited rotation angles, and the extremely complex distribution of interacting biomolecules within the cell.

To study structures in situ, the feature of interest must be identified and extracted from the tomogram. While specialized tools have been developed for identification of specific features, general-purpose cellular tomogram annotation remains an almost entirely manual task. This task is extremely laborintensive, requiring $\sim 1$ man-week for an expert to annotate of a typical tomogram [1]. With the increasing number of cellular tomograms being produced by automated methods, annotation has become the primary limiting factor in this CryoET data analysis.

Deep neural networks are now a popular method for processing images and other types of data. Indeed, these methods have become the standard method for tasks such as voice recognition and computervision applications [2]. The complex interconnections combined with nonlinearities introduced via the activation function in the network permits recognition of complicated features. We have developed a method based on convolutional neural networks, which is capable of learning a wide range of possible feature types. Trained on a small set of 2D samples given by an expert human annotator, the neural network can automatically annotate tomograms of the same sample taken in similar condition. This method can readily discriminate between subtle differences such as ribosome vs. RubisCOs, or mitochondrial membranes vs. other organelle membranes.

After annotating features of interest, the extracted subvolumes can be aligned and averaged to reduce noise and compensate for the missing wedge artifact, producing an intermediate resolution structure of the complex [3]. To resolve the heterogeneity among the extracted subvolumes, we present a dimension reduction technique based on deep auto-encoder that maps each subvolume to a point in a conformation space for further classification. Taking the advantage of deep neural networks, it is also capable of compensating the missing wedge artifact and extracting conformation changes that are non-linear to the intensity of the particles.

\section{References:}

[1] Hecksel, C. W., et al, Microscopy and Microanalysis, 22(03), (2016), 487.

[2] LeCun, Y., Bengio, Y., \& Hinton, G. Nature, 521(7553), (2015),436.

[3] Asano, S., et al, Science (New York, N.Y.), 347(6220), (2015), 439. 

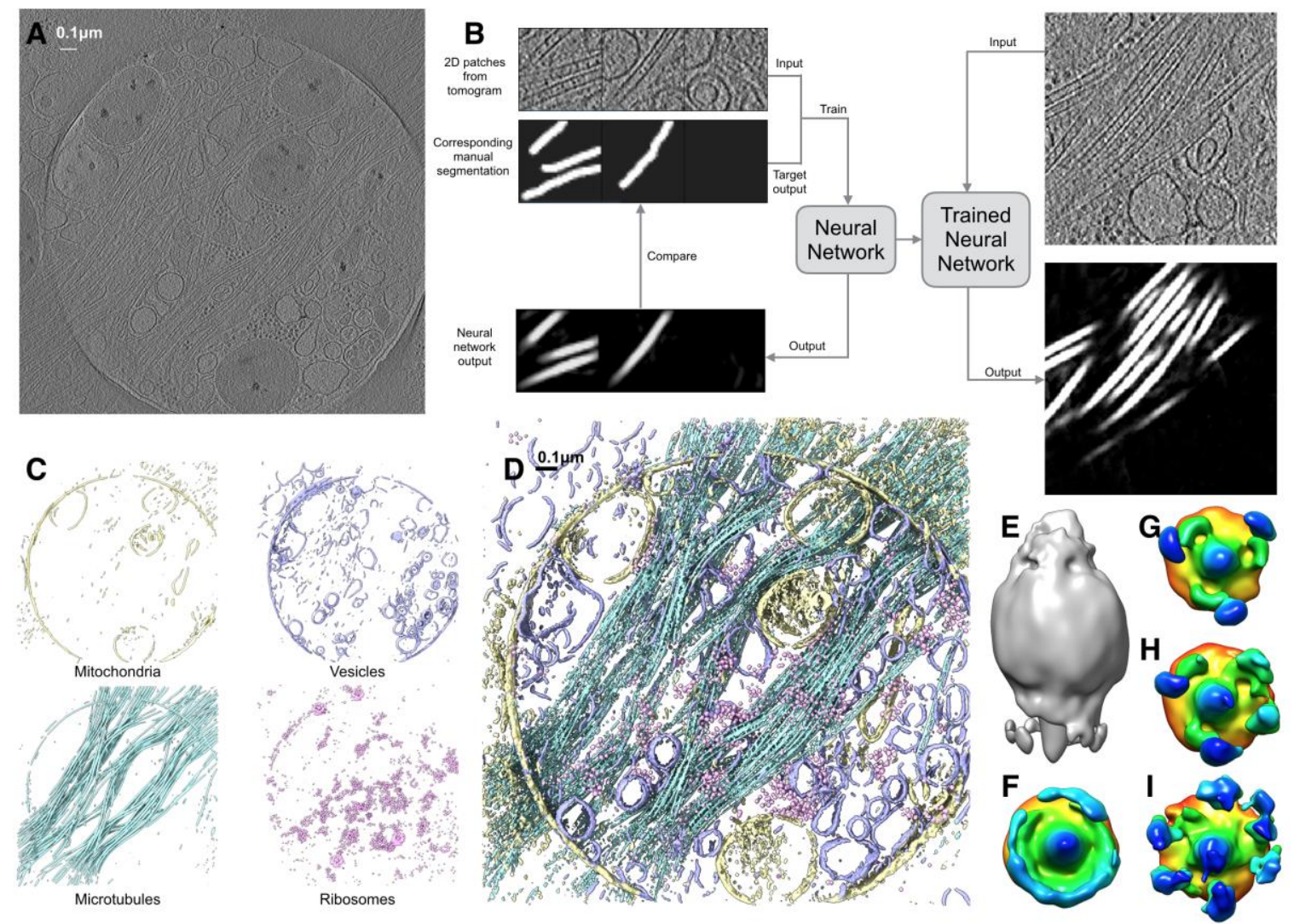

Figure 1. A. Raw tomogram of PC12 cell. B. Workflow of tomogram annotation. C. Annotation four features of interest in the PC12 tomogram. D. Combined result of PC12 tomogram annotation. (Best viewed in color.) E. Side view of a Syn5 phage subtomogram average. F. Bottom view of Syn5 subtomogram average, colored by height. The smeared out density around the phage tail is the average of tail fibers in different conformation. G-I: Classification result of Syn5, showing different poses of tail fibers. 